\title{
2 UNA PEDAGOGÍA ANTIMÉTODO. UNA PERSPECTIVA FREIRIANA
}

\author{
Donaldo Macedo
}

Universidad de Massachusetts, Boston

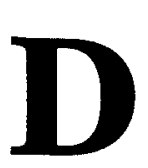

ebido a que el "modelo bancario" capitalista de la educación genera cada vez más fracaso, muchos pedagogos y muchas pedagogas liberales y neoliberales se están fijando en la pedagogía de Paulo Freire como alternativa. Ya no es posible argumentar que la pedagogía de Freire es únicamente apropiada para contextos tercermundistas. Por una parte, estamos observando la rápida "tercermundialización" de Norteamérica, donde las inner cities de muchas ciudades se asemejan cada vez más a los barrios de chabolas del Tercer Mundo por su elevado índice de pobreza, violencia, analfabetismo, explotación, personas "sin techo" y miseria. El abandono que sufren las inner cities y la perniciosa degeneración de sus infraestructuras, que incluye también sus escuelas, hace muy difícil mantener la división artificial entre Primer Mundo y Tercer Mundo. Es tan fácil encontrar tercermundismo en las inner cities del Primer Mundo como descubrir opulencia "primermundista" entre las oligarquías de El Salvador, Guatemala y tantos otros países del Tercer Mundo. La "tercermundialización" de las inner cities norteamericanas ha producido también fracasos educativos a gran escala que han generado índices de abandono escolar entre los estudiantes de las minorías que van del $50 \%$ de las escuelas públicas de Boston al $70 \%$ en áreas metropolitanas más grandes como la de Nueva York.

Ante este fenómeno de fracaso educativo, las educadoras y los educadores conservadores en general han ido hacia atrás en un intento de salvar su 
statu quo y frenar el proceso de "morenización" de Estados Unidos. Estas pedagogas y estos pedagogos conservadores han intentado apropiarse de nuevo del debate educativo y estructurar el discurso educativo en términos de privatización y de competencia entre colegios. El currículum oculto del movimiento que propone la privatización de las escuelas consiste en quitarles recursos a los colegios pobres que están al borde de la bancarrota para sostener a las escuelas privadas o adineradas. Se habla de la libertad de elegir una escuela privada, pero ésta es sólo privada en el sentido de que genera beneficios privados, aunque esté sostenida por fondos públicos. Lo que raramente se discute en el debate sobre el sistema escolar norteamericano es que los colegios públicos constituyen una parte esencial de la construcción de cualquier sociedad democrática. En realidad, las educadoras y los educadores conservadores llegan a reconocer que una sociedad democrática que elude su responsabilidad pública es una democracia en crisis. Una sociedad que equipara la privatización guiada por el lucro con la democracia es una sociedad que tiene unas prioridades confusas. Una sociedad democrática que cree, falsamente, a la vista de la debacle del sistema de ahorro y préstamos y de los escándalos de Wall Steet, que la calidad, la productividad, la honestidad y la eficiencia sólo pueden alcanzarse a través de una privatización que busque la rentabilidad, es una sociedad que demuestra una bancarrota de ideas éticas e intelectuales. Si creemos en la línea argumental que afirma que lo "privado" es lo mejor, deberíamos volver a considerar la cuestión que plantea Jack Beaty: “ ¿Privatizaríamos el Pentágono para mejorar nuestro sistema público de defensa?"' ¿Lograría erradicar la lógica de que "lo privado es lo mejor" los problemas todavía existentes en el ejército, que van desde el acoso sexual cada vez más extendido, hasta el pago de más de 600 dólares por una tapa de retrete o de miles de millones por aviones que no vuelan? Muchos y muchas estadounidenses encontrarían completamente absurda la privatización del Pentágono, y reclamarían que se le diera prioridad nacional a una defensa fuerte. En vez de desmantelar aún más la educación pública, deberíamos hacer de ella una prioridad pública nacional. Me atrevería incluso a afirmar que la salvaguarda de nuestra democracia depende en mayor medida de la creación de ciudadanos y ciudadanas inteligentes que no de bombas inteligentes.

En contraste con la noción de mercado que ha seguido la reforma escolar en los Estados Unidos, muchos pedagogos y pedagogas liberales y neoliberales han redescubierto a Freire como una alternativa a la educación de domesticación conservadora que equipara la ideología del libre mercado con la democracia. Parte del problema con algunos de estos educadores y educadoras pseudocríticos y pseudocríticas es que, en nombre de la pedagogía de la liberación, reducen las principales ideas de Freire a un método. Según Stanley Aronowitz, el fetichismo norteamericano por el método ha permitido que las

\footnotetext{
'Beaty, Jack, en el número de The Boston Globe del 14 de agosto de 1992.
} 
ideas filosóficas de Freire hayan sido "asimiladas con la obsesión dominante en la educación norteamericana, siguiendo una tendencia de todas las ciencias sociales y humanas, por los métodos de verificación del conocimiento y, en las escuelas, de la enseñanza, es decir, de la transmisión de conocimiento a estudiantes que de otro modo no estarían preparados" ${ }^{2}$.

Este fetichismo por el método dificulta perniciosamente la adhesión al pronunciamiento del propio Freire contra la importación y la exportación de la metodología. En el curso de una larga conversación que mantuve con él acerca de este tema, dijo lo siguiente: "Donaldo, no quiero ser importado o exportado. Es imposible exportar prácticas pedagógicas si no se reinventan. Por favor, diles a tus colegas pedagogos estadounidenses que no me importen. Pídeles que recreen y reescriban mis ideas".

Las principales ideas de Freire referidas al acto del saber trascienden los métodos por los que es conocido. De hecho, según Linda Birnbi, "la originalidad del trabajo de Freire no radica en la eficacia de sus métodos de alfabetización, sino, sobre todo, en la originalidad de sus contenidos, diseñados para desarrollar nuestra conciencia" 3 como parte de una pedagogía humanizadora. Según Freire, "una educación humanizadora es el camino a través del cual hombres y mujeres pueden tomar conciencia de su presencia en el mundo, de la manera en que ellos y ellas actúan y piensan cuando desarrollan todas sus capacidades, teniendo en cuenta sus necesidades pero también las necesidades y aspiraciones de los demás"4.

Dicho esto, ¿por qué algunos educadores, en su intento por cortar las cadenas de las prácticas educativas opresivas, abogan ciegamente por el modelo dialógico y crean, a su vez, una nueva forma de rigidez metodológica ligada a una opresión benévola, todo ello bajo la apariencia de democracia, con el solo propósito de que es por vuestro propio bien? Muchos y muchas de entre nosotros y nosotras hemos sido testigos de ciertos contextos pedagógicos en los que se nos requiere implícita o explícitamente que hablemos sobre nuestra experiencia como un acto de liberación. Todas y todos hemos asistido a conferencias donde el o la conferenciante es castigado o castigada porque deja de situarse a sí mismo dentro de la historia, es decir, no primó sus propias experiencias al tratar cuestiones de democracia crítica, sin importar si tenía cosas importantes y reveladoras que decir. Esto equivale a rechazar a Marx porque no nos hechizó con sus experiencias personales.

\footnotetext{
${ }^{2}$ Aronowitz, Stanley, “Paulo Freire's Radical Democratic Humanism”, en Peter McLaren \& Peter Leonard (eds.), Paulo Freire: A Critical Encounter, Londres: Routledge, 1993, p. 8.

${ }^{3}$ Birnbi, Linda, citado en Moacir Gadotti, Convite a Leitura de Paulo Freire, São Paulo: Editora Scipione, 1989, p. 32.

${ }^{4}$ Freire, Paulo, \& Frei Betto, Essa Iscola Chamada Vida, São Paulo: Atica, 1985, pp. 14-15.
} 
La apropiación del método dialógico como un proceso para compartir experiencias se reduce, a menudo, a una variante de la terapia de grupo que se centra en la psicología del individuo. A pesar de que algunos pedagogos y algunas pedagogas pueden aducir que este proceso genera una zona de confort pedagógico, en mi opinión, apenas va más allá de hacer sentirse bien a las personas oprimidas sobre su propio sentimiento de victimización. En otras palabras, el compartir experiencias no debería ser entendido tan sólo en términos psicológicos. Inevitablemente requiere también un análisis político e ideológico. Es decir, el compartir experiencias debe ser entendido siempre dentro de una praxis social que implique tanto la reflexión como la acción política. En resumen, debe implicar siempre un proyecto político dirigido a desmantelar las estructuras y los mecanismos opresivos.

Esta sobredosis de celebración experimental que caracteriza a algunas ramas de la pedagogía crítica ofrece una perspectiva reduccionista de la identidad y la experiencia dentro, y no fuera, de las problemáticas del poder, la agencia y la historia. Al abusar del legado y de la importancia de sus respectivas voces y experiencias, estos/as educadores/as no consiguen en muchas ocasiones ir más allá de una noción o de una diferencia estructurada en dicotomías polarizadoras y de una apelación acrítica al discurso de la experiencia ${ }^{5}$. Por esta razón, invocan una forma pedagógica romántica que da exotismo a las experiencias vividas como un proceso de hacerse voz. Al renunciar a relacionar las experiencias con la política cultural y con la democracia crítica, estos educadores reducen su pedagogía a una forma de narcisismo de clase media. Por un lado, el método dialógico provee a los y las participantes con un espacio de terapia de grupo donde exponer sus agravios y, por otro lado, ofrece al/la educador/a o dinamizador/a una zona pedagógica segura para tratar su culpabilidad de clase.

Al renunciar a tratar el tema de los privilegios de clase, el/la educador/a pseudocrítico/a proclama dogmáticamente la necesidad de facultar, de dotar de palabra a los y las estudiantes. A estos/as educadores/as les llega a traicionar su propio lenguaje. En vez de crear estructuras pedagógicas que harían posible que los estudiantes oprimidos se dotasen de poder, proclaman de manera paternalista que "necesitamos dotar de poder a los y las estudiantes". Esta postura conduce a menudo a la creación de lo que defino como proxenetas de la alfabetización y de la pobreza hasta el punto de que, aunque proclaman la necesidad de dotar de poder a los y las estudiantes, están en realidad reforzando sus propias posiciones privilegiadas.

El siguiente ejemplo clarificará mi afirmación: una colega progresista que había estado trabajando conmigo en un proyecto de alfabetización comuni-

"Giroux, Henry, "The Politics of Difference and Multiculturalism in the Era of the Los Ángeles Uprising", en el Journal of the Midwest Modern Language Association [pendiente de publicación]. 
taria traicionó su discurso liberal de dotar de poder a la comunidad cuando una de las agencias con las que trabajamos solicitó mi ayuda para redactar una propuesta de alfabetización matemática para ellos. Yo estuve de acuerdo y agradecí la oportunidad. Una de mis metas es desarrollar estructuras para que los miembros y las agencias de una comunidad puedan tomar la iniciativa y diseñar su propio curso de acción, eliminando de esta forma la necesidad de seguir contando con nuestra presencia y nuestro asesoramiento continuados. En otras palabras, nuestro éxito en la creación de estructuras para que los miembros de una comunidad se doten de poder a sí mismos reside en el grado en que nuestra presencia y conocimiento experto dejen de ser necesarios para la comunidad, porque los miembros de dicha comunidad ya hayan adquirido su propio conocimiento experto, evitando así una especie de neocolonialismo.

Cuando mi colega se enteró de la propuesta de alfabetización matemática, se mostró reticente pero no se opuso abiertamente. Sin embargo, unas semanas después, cuando se enteró de que la subvención para alfabetización matemática que yo estaba elaborando con los miembros de la comunidad competía con nuestra propia propuesta desde la universidad, que había sido diseñada para proveer formación alfabetizadora a esos propios miembros de la comunidad, mi colega reaccionó casi de modo irracional. Adujo que la organización comunitaria que había redactado la concesión no había seguido un proceso democrático porque no la había implicado a ella en el desarrollo de la concesión. Según su visión, un proceso democrático y participativo se refería a la condición de que ella debía tomar parte en la acción comunitaria, a pesar de que ella no es miembro de la comunidad en concreto para la que había sido diseñada la concesión de alfabetización matemática. Aparentemente, en su opinión, una persona puede ser dotada de poder siempre y cuando esta toma de poder no usurpe los privilegios de los y las "expertos/as", su posición de poder. Ésta es una posición de poder diseñada para dotar de poder a otros de forma paternalista.

Cuando señalé las obvias contradicciones ideológicas en el comportamiento de mi colega, su respuesta fue rápida, agresiva y prácticamente automática: "me enfadaría mucho si consiguieran su propuesta y nosotros no consiguiéramos la nuestra". Para mí quedó muy claro que el compromiso político real de mi colega con la comunidad dependía de la medida en que su posición de "experta" no se viera amenazada. Es decir, el "experto" alfabetizador benefactor, una persona contraria al orden establecido, se asegura de que su posición privilegiada dentro de ese orden como "experto" contrario a ese orden nunca sea absorbida por los miembros de una comunidad dotados de poder.

Esta actitud colonizadora, paternalista, llevó a esta misma colega a manifestar públicamente, en un importante congreso, que la gente de la comunidad no necesita ir a la universidad porque, como saben mucho más que los miembros de la comunidad universitaria, poco puede enseñarles ésta. A la 
vez que hacía tales declaraciones públicas, esta colega se encontraba muy atareada mudándose desde una inner city hasta un suburbio adinerado para asegurarse de que sus hijos pudieran ir a escuelas mejores.

Una actitud similar surgió en una reciente reunión para desarrollar una propuesta de ayuda para la relación entre comunidad y universidad. Durante el encuentro, una profesora liberal blanca protestó con razón contra la ausencia de miembros de la comunidad en el comité. Sin embargo, en un intento de revalorizar la base de conocimiento de la comunidad, no tardó en caer en un paternalismo romántico al afirmar que las personas de la comunidad sabían mucho más que los profesores universitarios y que deberían ser ellas las que fueran invitadas para venir a enseñarnos, en lugar de enseñarles nosotros/as a ellas. Esta postura no sólo desanima a los miembros de la comunidad a la hora de acceder al capital cultural del cual estos profesores de universidad se han beneficiado ampliamente, sino que desdibuja el contexto real que hace del capital cultural universitario algo indispensable para cualquier auténtico tipo de toma de poder. También huele a la falsa generosidad del paternalismo al que Freire se oponía acérrimamente:

La pedagogía de los oprimidos animada por un auténtico humanismo (no árido humanitarismo) se presenta generosamente a sí misma como una pedagogía del hombre, una pedagogía que comienza con los egoístas intereses de los opresores (un egoísmo envuelto en falsa generosidad paternalista) y hace de los oprimidos objetos de su humanitarismo, y que por sí misma mantiene y representa la opresión. Es un instrumento de deshumanización ${ }^{6}$.

La actitud pedagógica paternalista representa un cierto narcisismo de clase media que da origen a pedagogos/as pseudocríticos/as que son parte del mismo enfoque instrumentista de la alfabetización al que dicen renunciar. $\mathrm{El}$ enfoque instrumentista de la alfabetización no hace referencia únicamente al objetivo de crear lectores que cumplan los requisitos básicos de nuestra sociedad contemporánea, como proponen las educadoras y educadores conservadores. La alfabetización instrumentista incluye también los más altos niveles de alfabetización a través de la especialización disciplinar y de la hiperespecialización. Las pseudoeducadoras y los pseudoeducadores son parte de esta alfabetización instrumental en la medida en la que reducen el enfoque dialógico de Freire a una forma de especialización. En otras palabras, tanto la alfabetización instrumental para los pobres desde una perspectiva basada en la competencia y en la acumulación bancaria de habilidades, como la más elevada de las formas de alfabetización instrumental para los ricos, adquirida a través de la universidad en forma de especialización profesional, tienen un factor en común: ambos enfoques evitan el desarrollo

\footnotetext{
' Freire, Paulo, Pedagogy of the Oppressed, Nueva York: Continuum Publication, 1990, p. 39.
} 
de un pensamiento crítico que le permita a uno interpretar el mundo de manera crítica y entender los porqués y las conexiones que existen detrás de los hechos. La aproximación instrumentalista a la alfabetización, incluso al más alto nivel de especialización (incluyendo el método como una forma de especialización), funciona para domesticar la conciencia a través de una constante desarticulación entre la lectura reduccionista y estrecha del área de especialidad de cada uno y la lectura del universo dentro del cual se sitúa la especialización de cada uno. Esta incapacidad para ligar la lectura de la palabra con el mundo, si no es combatida, acabará debilitando las ya de por sí débiles instituciones democráticas y las injustamente asimétricas relaciones de poder que caracterizan la naturaleza hipócrita de las democracias contemporáneas. En los niveles más bajos de la alfabetización instrumental, una persona semianalfabeta lee las palabras pero es incapaz de leer el mundo. En los más altos niveles de alfabetización instrumental adquirida a través de la especialización, se puede ser ignorante de las demás áreas del saber que constituyen el mundo del conocimiento. Este especialista semianalfabeto fue definido por Ortega y Gasset como un "ignorante educado". Es decir, "no es educado, porque formalmente ignora todo aquello que no entre dentro de su especialidad; pero tampoco es ignorante porque es un 'científico' y 'conoce' muy bien su diminuta porción del universo"?

Debido a que el "ignorante educado" se ocupa principalmente de su diminuta porción del mundo, desconectada de otros cuerpos de conocimiento, nunca será capaz de relacionar el flujo de información hasta el punto de adquirir una lectura crítica del mundo. Una lectura crítica del mundo implica, según Freire, "una comprensión dinámica entre la sensibilidad del mundo menos coherente y una comprensión más coherente del mundo". Esto explica la incapacidad de, por ejemplo, los especialistas médicos de los Estados Unidos, quienes han contribuido a importantes avances tecnológicos en la medicina, para entender o valorar por qué más de 30 millones de estadounidenses no tienen acceso a esta tecnología médica y por qué tenemos todavía los índices de mortalidad infantil más altos en comparación con los de otras naciones desarrolladas.

Finalmente, acabo este artículo proponiendo una pedagogía antimétodo que rechace la rigidez de los modelos y paradigmas metodológicos. La pedagogía antimétodo nos obliga a entender el diálogo como una forma de praxis social para que el compartir experiencias sea un hecho guiado por la reflexión y la acción política. El diálogo como praxis social "implica que el rescate de la voz de los oprimidos sea una condición fundamental para la emancipación humana"9.

\footnotetext{
' Ortega y Gasset, José, The Revolt of the Masses, Nueva York: Norton, 1932, p. 112.

${ }^{8}$ Freire, Paulo, \& Macedo, Donaldo, Literacy: Reading the Word and the World, South Hadley, MA: Bergin \& Garvey, 1987, p. 131.

'Aronowitz, Stanley, "Paulo Freire's Radical Democratic Humanism", p. 18.
} 
La pedagogía antimétodo nos libera también del habitual camino de las certezas y las especializaciones. Rechaza la mecanización del intelectualismo. En resumen, invoca la iluminación que ofrecen las principales ideas de Freire para guiarnos hacia el camino crítico de la verdad, hacia la recuperación de nuestra dignidad en peligro, hacia la reivindicación de nuestra humanidad. Nadie podría argumentar mejor contra la reducción del diálogo y el planteamiento de problemas a un mero método como el propio Freire:

La educación basada en el planteamiento de problemas es la futuridad revoiucionaria. Luego es profética... Luego corresponde a la naturaleza histórica del hombre. Luego confirma a los hombres como seres que se trascienden a sí mismos. Luego se identifica con el movimiento que compromete a los hombres como seres conscientes de su carácter inconcluso, un movimiento histórico que tiene su punto de partida, sus sujetos y su objetivo"10.

La pedagogía del antimétodo no se adhiere tan sólo a la visión de Freire de la educación como futuridad revolucionaria; también celebra la elocuencia del poema de Antonio Machado: "Caminante no hay camino, se hace camino al andar".

\section{RESUMEN}

Ante el fracaso educativo, algunos educadores liberales o neoliberales han redescubierto a Freire como una alternativa a un modelo educacional más conservador. Han reivindicado la pedagogía de Freire al mismo tiempo que una actitud paternalista hacia aquellos estudiantes o comunidades que intentaban dotarse a si mismos de poder. Estos educadores y educadoras han intentado también estructurar el debate educativo en base a la competitividad y privatización de las escuelas. Han reducido la pedagogía de Freire al método, sin tener en cuenta el hecho de que las ideas principales de Freire trascienden el mero método para guiarnos hacia el pensamiento crítico y hacia una toma de conciencia sobre nuestra presencia en el mundo. El método dialógico, entendido como solamente un método, puede consistir en nada más que un mero grupo de terapia. En cambio, si vamos más allá de su contenido metodológico, el diálogo freiriano implica todo un proyecto político dirigido a desmantelar las estructuras opresivas.

\footnotetext{
${ }^{10}$ Freire, Paulo, citado en Stanley Aronowitz, "Paulo Freire's Radical Democratic Humanism",
} pp. 11-12. 
La pedagogía antimétodo rechaza los rígidos paradigmas metodológicos y nos hace entender el diálogo como una forma de praxis social. En este sentido, el compartir experiencias está enfocado a la reflexión y la acción política. La pedagogía antimétodo nos libera de certezas y especializaciones y estimula nuestra capacidad crítica para entender el mundo y hacernos conscientes de nuestra presencia en él.

Palabras clave: Método dialógico, antimétodo, praxis social, diálogo freiriano.

\section{ABSTRACT}

Against educational failure, some liberal or neoliberal educators have rediscovered Freire as an alternative to a more conservative educational model. They have claimed Freire's pedagogy while adopting a paternalistic attitude towards those students or communities that try to empower themselves. These educators have also attempted to structure the educational debate in terms of school competition and privatization. They have reduced Freire's pedagogy to a method without taking into account the fact that Freire's leading ideas transcend a mere method to guide us towards critical thinking and towards the awareness of our own presence in the world. The dialogic method, if taken only as a method, can consist of no more than a mere group therapy. Yet if we move beyond its methodological content, Freirean dialogue involves a whole political project that aims to dismantle oppressive structures.

The antimethod pedagogy refuses rigid methodological paradigms and makes us view dialogue as a form of social praxis. In this way, the sharing of experiences is aimed at reflection and political action. The antimethod pedagogy frees us from certainties and specialisms and fosters our critical capability to read the world and become aware of our presence in it.

Key words: Dialogic method, antimethod, social praxis, Freirean dialogue. 